\title{
Bosutinib as a fourth-line therapy for a patient with T315I-positive lymphoid blastic phase chronic myeloid leukemia: A case report
}

\author{
YUKIKO KOMENO $^{1}$, NAOYUKI UCHIDA ${ }^{2}$, YUMIKO SATOH ${ }^{3}$, HIDEKI URYU $^{1}$, YUKO IWATA ${ }^{1}$, \\ AKIKO MASUDA $^{3}$, KUNIKO IIHARA ${ }^{4}$, YUTAKA YATOMI ${ }^{3}$, SHUICHI TANIGUCHI $^{2}$ and TOMIKO RYU ${ }^{1}$ \\ ${ }^{1}$ Department of Hematology, Japan Community Healthcare Organization (JCHO) Tokyo Yamate Medical Center, \\ Hyakunin-cho, Shinjuku, Tokyo 169-0073; ${ }^{2}$ Department of Hematology, Toranomon Hospital, Toranomon, Minato, \\ Tokyo 105-8470; ${ }^{3}$ Department of Clinical Laboratory, The University of Tokyo Hospital, Hongo, Bunkyo, Tokyo 113-8655; \\ ${ }^{4}$ Department of Pathology, JCHO Tokyo Yamate Medical Center, Hyakunin-cho, Shinjuku, Tokyo 169-0073, Japan
}

Received October 14, 2015; Accepted February 17, 2017

DOI: $10.3892 / \mathrm{ol} .2017 .5989$

\begin{abstract}
A 35-year-old male was diagnosed with chronic myeloid leukemia in the chronic phase and was prescribed $100 \mathrm{mg}$ daily dasatinib. However, dasatinib was discontinued due to thrombocytopenia, and within six months, the disease progressed to the lymphoid blastic phase. Hyper-cyclophosphamide, vincristine, adriamycin and dexamethasone chemotherapy combined with $140 \mathrm{mg}$ dasatinib or $600 \mathrm{mg}$ imatinib was prescribed. The two inhibitors were soon discontinued due to severe thrombocytopenia and jaundice, respectively. Myelosuppression persisted subsequent to the nadir. Bone marrow (BM) aspiration and biopsy revealed hypercellular marrow filled with blasts. Sequencing of the leukemia cells revealed overlapping peaks for the wild-type sequence and the T315I mutant sequence. The patient was treated with $500 \mathrm{mg}$ bosutinib (which was later reduced to $300 \mathrm{mg}$ ) for pretransplant cytoreduction. After 5 months, the patient's spleen exhibited a reduction in volume and the percentage of blasts in the BM decreased from 96.1 to $17.5 \%$. The patient successfully underwent cord blood transplantation. The patient has been disease-free for 5 months subsequent to transplantation. This case suggests that bosutinib may be effective for cytoreduction prior to stem cell transplantation, unless the leukemia cells consistently harbor the T315I mutation.
\end{abstract}

\section{Introduction}

Chronic myeloid leukemia (CML) is a myeloproliferative disorder that arises in hematopoietic stem cells (1). It is

Correspondence to: Dr Yukiko Komeno, Department of Hematology, Japan Community Healthcare Organization (JCHO) Tokyo Yamate Medical Center, 3-22-1 Hyakunin-cho, Shinjuku, Tokyo 169-0073, Japan

E-mail: ykomeno-tky@umin.ac.jp

Key words: chronic myeloid leukemia, blastic phase, tyrosine kinase inhibitor, bosutinib, T315I mutation characterized by a reciprocal $t(9 ; 22)$ translocation that leads to the formation of the Philadelphia chromosome, which in turn produces the BCR-ABL1 fusion protein. BCR-ABL1 is a constitutively active tyrosine kinase, which transmits proliferation and survival signals through the Src family kinases, Lyn and Hck, to the downstream targets, STAT5 and Ras/ERK $(2,3)$.

CML has a triphasic clinical course (1). In the chronic phase (CP), excessive proliferation of mature myeloid cells occurs (1). In the accelerated phase (AP), CML cells accumulate chromosomal and genetic abnormalities, and blasts in the peripheral blood (PB) or bone marrow (BM) increase to $10-20 \%$ (1). Eventually, the disease progresses to the blastic phase (BP), where blasts account for $>20 \%$ of the cells in the PB or BM (1). In $~ 70 \%$ of BP cases the blast lineage is myeloid, whereas in the remaining 20-30\% of BP cases the lineage is lymphoid (1).

Tyrosine kinase inhibitors (TKIs) have markedly improved the prognosis of patients with CML. Currently, five different TKIs are available for the treatment of CML: Imatinib, nilotinib, dasatinib, bosutinib and ponatinib (4). Imatinib, nilotinib and dasatinib are approved for first-line treatment of CML, and bosutinib and ponatinib are available for resistant and intolerant CML patients. As of July 2015, ponatinib is not yet available in Japan. Secondary resistance to TKIs occurs in $20-30 \%$ of patients with CML, and is mainly due to mutations in the $A B L 1$ kinase domain $(5,6)$. The most intractable mutation is the T315I gatekeeper mutation, which potently interferes with the binding of TKIs to BCR-ABL1 (7). Of the five TKIs, only ponatinib is active against the T315I mutant (4,8-11). Stem cell transplantation is currently reserved for patients with CML-AP/BP and selected cases of CML-CP $(4,12,13)$.

Bosutinib is a dual Src and ABL1 tyrosine kinase inhibitor (14-21). It is active against various BCR-ABL1 mutations, including those associated with imatinib, dasatinib and nilotinib resistance. However, bosutinib is not active against the T315I and V299L BCR-ABL1 mutations $(4,10,11)$. Bosutinib inhibits Src family kinases, including Src, Lyn, Fgr and Hck (14). However, unlike other TKIs, bosutinib has exhibited minimal inhibitory activity against platelet-derived growth factor receptor and c-KIT (14). Therefore, bosutinib has a distinct 
toxicity profile compared to other TKIs (15-21). The present case study provides a report of a patient with T315I-positive CML-BP who was successfully treated with bosutinib as a fourth-line therapy prior to cord blood transplantation.

\section{Materials and methods}

Sequencing of the ABL1 kinase domain of BCR-ABL1. Total RNA was extracted from total BM cells using ISOGEN (Nippon Gene, Tokyo, Japan) and $1 \mu \mathrm{g}$ from each sample was reverse transcribed using a Transcriptor 1st Strand cDNA Synthesis kit (Roche Diagnostics, Tokyo, Japan). The ABL1 tyrosine kinase domain of $B C R-A B L 1$ was amplified with nested RT-PCR with the following primers: 1st forward, (designed on $B C R$ gene), 5'-TTCAGAAGCTTCTCCCTGCAT-3' and reverse (located in $A B L$ gene), 5'-CTTCGTCTGAGATAC TGGATTCCT-3'; 2nd forward (located in $A B L$ gene), 5'-AAG CGCAACAAGCCCACTGTCTAT-3' and reverse (located in $A B L$ gene), 5'-CTTCGTCTGAGATACTGGATTCCT-3'. Amplified fragments were directly sequenced using an ABI PRISM 310 Genetic Analyzer (Applied Biosystems; Thermo Fisher Scientific, Inc., Waltham, MA, USA). Written informed consent was obtained from the patient in the present study, according to the Declaration of Helsinki.

Staining.BM aspirate smears were stained with May-Grünwald's and Giemsa's staining solution. Images were acquired at room temperature using a BX50 light microscope (Olympus Corporation, Tokyo, Japan) equipped with a DS-Fi2 camera, Digital Sight DS-U3 controller and NIS-Elements D software version 3.20 (all Nikon Corporation, Tokyo, Japan). BM biopsy samples were fixed in $10 \%$ neutral buffered formalin solution, decalcified in Osteosoft (Merck KGaA, Darmstadt, Germany), sectioned (3- $\mu \mathrm{m}$ thick), and stained with hematoxylin and eosin. Images were acquired at room temperature using a BX53 light microscope equipped with DP21 camera/controller (both Olympus Corporation).

\section{Case report}

A previously healthy 35-year-old Japanese male visited the Department of Surgery for a left inguinal hernia in March 2014. The patient had mild hepatomegaly and marked splenomegaly. His blood cell counts were: White blood cells (WBC) $345,820 / \mu 1$ (blasts $4.0 \%$, promyelocytes $0.0 \%$, myelocytes $24.5 \%$, metamyelocytes $3.5 \%$, band $16.5 \%$, segmented $44.5 \%$, monocytes $1.0 \%$, lymphocytes $1.0 \%$, eosinophils $2.0 \%$, basophils $3.0 \%$ ), hemoglobin $7.3 \mathrm{~g} / \mathrm{dl}$ and platelets $113 \times 10^{3} / \mu 1$. The following day, the patient was referred to the Department of Hematology. BM aspiration and biopsy revealed hypercellular marrow with $9.4 \%$ blasts (Fig. 1A) and the karyotype was 46,XY, $t(9 ; 22)(q 34 ; q 11.2)[20 / 20]$. The patient was diagnosed with CML-CP. However, the disease rapidly progressed to AP (platelets $65 \times 10^{3} / \mu 1$ ). The patient was hospitalized in April 2014, and $100 \mathrm{mg}$ dasatinib was prescribed. WBC and platelet counts normalized, while hemoglobin levels increased. The splenomegaly was also reduced from 8 to $3.5 \mathrm{~cm}$ below the umbilicus. On the 9th day of hospitalization, the patient was discharged. The patient continued to take dasatinib until his platelet count lowered to $49 \times 10^{3} / \mu 1$, when dasatinib was
A

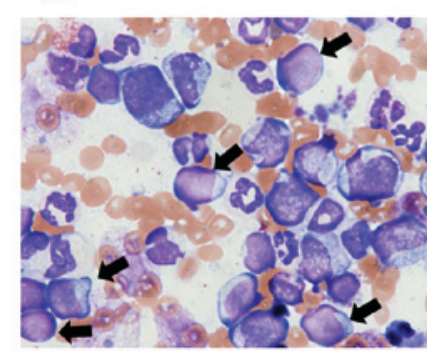

B

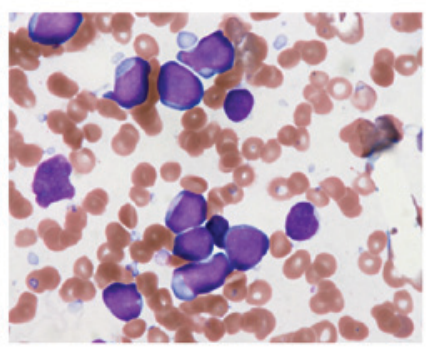

Figure 1. May-Grünwald Giemsa staining of BM aspirate smears. (A) BM at the initial diagnosis of CML-CP. Black arrows illustrate the blasts. (B) BM at the diagnosis of CML-BP. Original magnification, $\mathrm{x} 400$. BM, bone marrow; $\mathrm{CML}$, chronic myeloid leukemia; $\mathrm{CP}$, chronic phase; $\mathrm{BP}$, blastic phase.

A

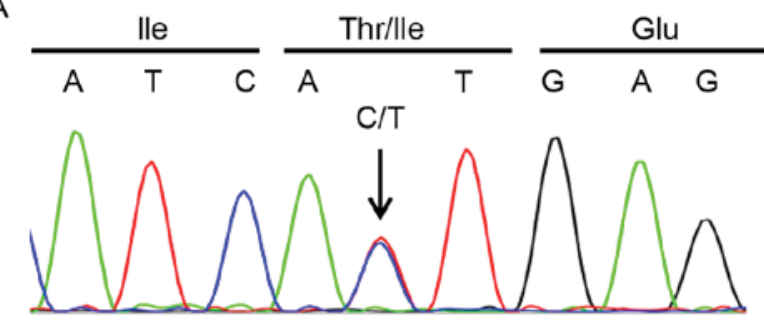

B

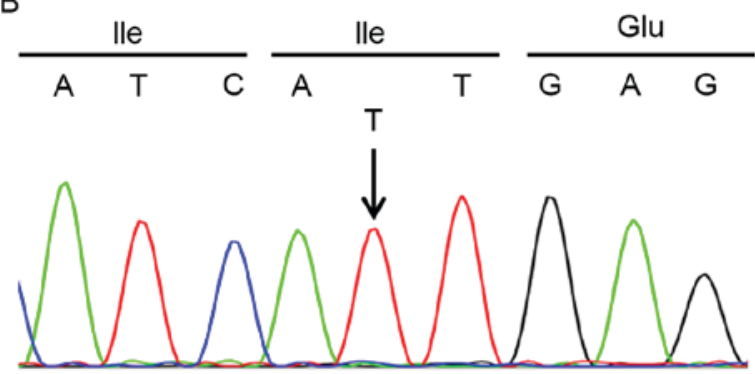

Figure 2. Sequencing chromatograms of the $B C R-A B L 1$ fusion gene, particularly the regions flanking amino acid T315 of $A B L 1$. (A) Sequencing chromatogram that was obtained prior to bosutinib administration. The central codon, ACT, corresponds to wild-type T315. The overlapping peaks of wild-type and $\mathrm{C}>\mathrm{T}$ (T315I) mutation $A B L 1$ are indicated with an arrow. (B) Sequencing chromatogram that was obtained four months subsequent to bosutinib administration. Only the T315I peak (ATT, indicated with an arrow) was detected.

discontinued one month subsequent to the initial prescription. One month later, administration of dasatinib was resumed. Subsequent to this visit, no contact could be made with the patient.

In September 2014, the patient was hospitalized due to active nasal bleeding. Blood test results revealed levels of: WBC 3,790/ $\mu 1$, hemoglobin $7.6 \mathrm{~g} / \mathrm{dl}$ and platelets $8 \times 10^{3} / \mu 1$. $\mathrm{BM}$ aspiration and biopsy revealed hypercellular marrow with peroxidase-negative blasts $(85.5 \%$, Fig. 1B) characterized as $\mathrm{CD} 10^{+} / \mathrm{CD} 19^{+} / \mathrm{CD} 20^{-} / \mathrm{CD} 34^{+} / \mathrm{CD}^{\mathrm{dim}}$. Cytogenetic analysis revealed a Philadelphia chromosome with additional aberrations: 46, XY, t(9;22)(q34;q11.2), del(15)(q?)[6/20], 47,idem, $+\operatorname{der}(22) \mathrm{t}(9 ; 22)[2 / 20], 50$, idem $,+\mathrm{X}, \operatorname{del}(13)(\mathrm{q} ?),+14,+21$, $+\operatorname{der}(22) \mathrm{t}(9 ; 22)[3 / 20], 46, \mathrm{XY}[8 / 20]$. A diagnosis of CML B-lymphoid BP was made. Hyper-CVAD (cyclophosphamide, vincristine sulfate, adriamycin, dexamethasone) chemotherapy 


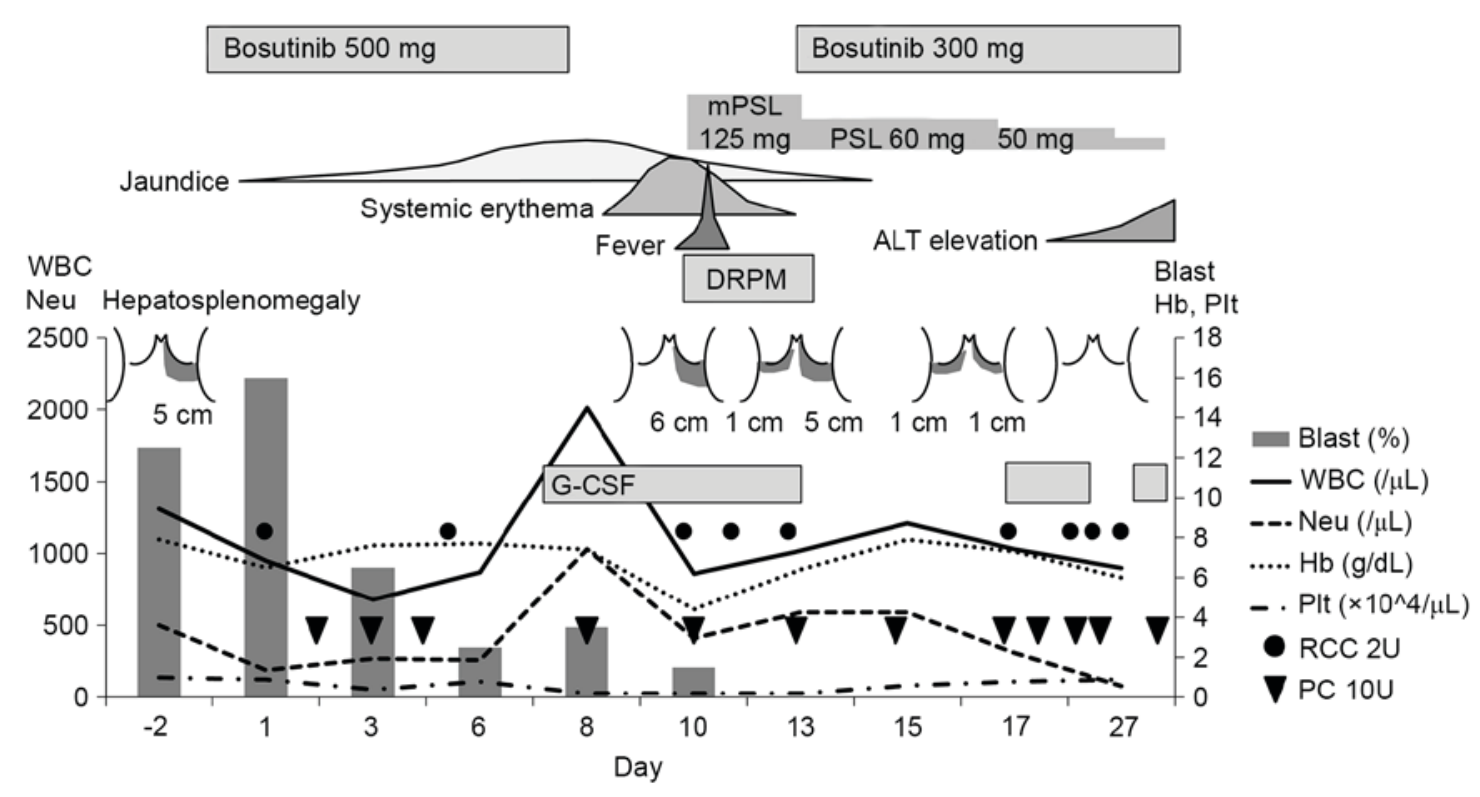

Figure 3. Summary of the clinical symptoms, blood analyses, and course of treatment for the present case report. Hepatosplenomegaly is indicated according to the size below the costal arches. mPSL, methylprednisolone; ALT, alanine transaminase; WBC, white blood cells; DRPM, doripenem; Neu, neutrophils; Hb, hemoglobin; Plt, platelets; G-CSF, granulocyte-colony stimulating factor (filgrastim); RCC 2U, 2 units of red cell concentrates; PC 10U, 10 units of platelet concentrates.
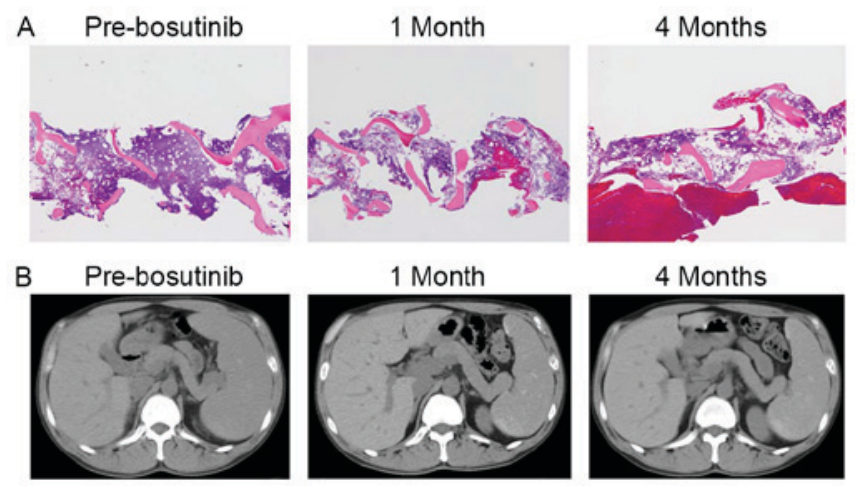

4 Months

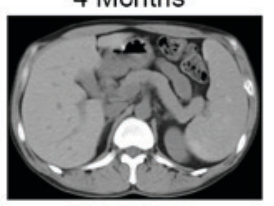

Figure 4. The patient's leukemia load was reduced with administration of bosutinib. (A) Hematoxylin and eosin staining of bone marrow biopsy samples that were collected prior to and 1 and 4 months subsequent to the administration of bosutinib. A reduction in cellularity was observed with time. Original magnification, x40. (B) Plain abdominal computed tomography scans were obtained prior to and 1 and 4 months subsequent to administration of bosutinib. Spleen volume was reduced following one month of bosutinib treatment. For the next three months, this reduced spleen volume was maintained.

was started in combination with $140 \mathrm{mg}$ dasatinib (22). The patient's platelet counts remained as low as $10 \times 10^{3} / \mu 1$, despite frequent transfusions. When dasatinib induced thrombocytopenia in the patient by day 7 , it was discontinued and the patient was switched to $600 \mathrm{mg}$ imatinib. Active nasal bleeding persisted even following cauterization. Therefore, dexamethasone was reduced from 8 doses to 5 doses to reduce mucosal toxicity. When the bilirubin and alkaline phosphatase levels reached $3.7 \mathrm{mg} / \mathrm{dl}$ and 1,012 IU/1, respectively, by day 16, imatinib was discontinued. However, pancytopenia persisted, and when BM aspiration and biopsy was performed on day 37, the blast level was $81.4 \%$. Considering the high leukemia burden of the patient, allogeneic stem cell transplantation was considered to be a high risk treatment $(4,12,13)$. Therefore, the patient underwent follow-up care in an outpatient setting, with transfusions performed 2-3 times per week.

In December 2014, the patient was re-admitted for leukemia debulking with bosutinib. BM aspiration and biopsy revealed hypercellular BM filled with blasts (96.1\%). Cytogenetic analysis revealed 46,XY,t(9;22)(q34;q11.2),del(15)(q?)[18/19] and 50, idem $,+X, \operatorname{add}(1)(\mathrm{p} 11), \operatorname{add}(13)(\mathrm{q} 12),+14,+21,+\operatorname{der}(22)$ $\mathrm{t}(9 ; 22)[1 / 19]$. Quantitative PCR detected major BCR-ABL1 at $3.1 \times 10^{5} \mathrm{copy} / \mu \mathrm{g}$ RNA. The T315I mutation was detected in the major $B C R-A B L 1$ sequencing. In the sequencing chromatogram, peaks of the wild type allele were revealed to overlap with the T315I mutant allele (Fig. 2), suggesting that almost half of the leukemia cells lacked the T315I mutation. Administration of bosutinib at $500 \mathrm{mg}$ per day was prescribed (Fig. 3). Notably, blasts in the PB, as well as hepatosplenomegaly, disappeared within 2 and 3 weeks, respectively. Adverse effects experienced by the patient included diarrhea [CTCAE v4.0 Grade (G) 2], anorexia (G3), jaundice (G3, bilirubin $4.6 \mathrm{mg} / \mathrm{dl})$, systemic erythema (G2) and papilloedema (G1). Bosutinib was discontinued on day 8 due to jaundice, yet was resumed at a dose of $300 \mathrm{mg}$ on day 14. This lower dose was better tolerated, however the levels of alanine transaminase (ALT) became elevated (G3, 187 IU/1), which required temporary interruption. The patient was referred to a collaborating hospital for transplantation in February 2015. After two months of bosutinib prescription, the blast level was decreased to $19.4 \%$, and major $B C R-A B L 1$ level was $3.5 \times 10^{4} \mathrm{copy} / \mu \mathrm{g}$ RNA. The patient continued to take bosutinib while waiting for the transplantation. After four months of bosutinib prescription, the patient's BM became more hypocellular (Fig. 4A) and his spleen did not show regrowth (Fig. 4B). Blasts in the BM were reduced to $17.5 \%$, and a cytogenetic analysis revealed a normal karyotype (46, XY [20/20]). Sequencing of $A B L 1$ of the BM sample 
only detected the T315I mutant allele (Fig. 2B). Stem cell transplantation was still considered necessary due to persistent pancytopenia and transfusion dependence, despite the absence of disease progression (based on the percentage of blasts in the PB or splenomegaly status). Subsequent to taking bosutinib for five months, the patient underwent cord blood transplantation at the end of April 2015 that was accompanied by high-dose cytarabine ( $2 \mathrm{~g} \mathrm{x} 2 /$ day for two days) followed by a conditioning regimen of fludarabine $\left(180 \mathrm{mg} / \mathrm{m}^{2}\right)$, an intravenous injection of busulfan $(12.8 \mathrm{mg} / \mathrm{kg})$ and melphalan $\left(80 \mathrm{mg} / \mathrm{m}^{2}\right)$. Engraftment was confirmed on day 25 . BM aspiration was performed on day 36 . The blast count was $0 \%$, the karyotype was 46,XX [20/20] (as a result of a female donor), and $B C R-A B L 1$ was not detected by quantitative PCR. Subsequent to becoming transfusion-independent, the patient was discharged from the hospital on day 64. Acute graft-vs.-host disease (GVHD) was observed in skin (stage I), but not in the liver (stage 0 ) or gut (stage 0 ) (Grade I). Major $B C R-A B L 1$ level in PB was $<0.0018 \%$ by International Scale at one month and two months subsequent to transplantation. Tacrolimus for GVHD treatment was discontinued at four months. At five months, the patient has no sign of relapse, and follow up was begun in an outpatient setting.

\section{Discussion}

Point mutations in the $A B L 1$ kinase domain have been associated with resistance to TKIs in patients with CML $(4-6,10,11)$. In the present case report, a patient with T315I mutation-positive CML-BC demonstrated a response to bosutinib over five months, and successfully underwent cord blood transplantation. Initially, when exhibiting CML-CP, the patient was intolerant to treatment with dasatinib [due to thrombocytopenia and low compliance (23). In addition, the patients CML-BP disease was resistant to chemotherapy combined with the TKIs, dasatinib or imatinib (22). Correspondingly, the patient was revealed to carry the T315I mutation. It has been reported that bosutinib is ineffective against T315I-mutated BCR-ABL1 in vitro $(4,10,11)$. In addition, in phase 1 and 2 clinical trials, CML-CP patients with the T315I mutation exhibited poor responses compared to those without the mutation (15-17). In Japan, bosutinib became available in December 2014 (21), whereas ponatinib $(8,9)$ and the non-TKI, omacetaxine $(24)$, which have been shown to be active against T315I-positive cases, were not available at this time. In the present case, the sequencing chromatogram of $A B L 1$ implied that at least half of the leukemia cells carried wild-type $B C R-A B L 1$ (Fig. 2A). Indeed, bosutinib was effective in debulking the leukemia cells in the patient. Furthermore, $A B L 1$ sequencing subsequent to the administration of bosutinib detected only the T315I peak (Fig. 2B). Thus, the present results demonstrate that when a patient carries cells with the T315I mutation, sequencing chromatograms of $A B L 1$ can potentially provide an estimate of the T315I-positive leukemia cell burden. Additionally, in cases where resistance/intolerance to multiple TKIs is a factor, bosutinib may potentially provide an effective pre-transplant therapy while promising new drugs including ponatinib $(8,24)$, omacetaxine (24) and axitinib (25) that target the T315I mutation are becoming widely available.
Furthermore, for individual situations, certain drugs may or may not be suitable due to preexisting comorbidities and/or pretreatments.

It has been reported that Src family kinases, including Lyn, Hck, and Fgr, perform critical roles in BCR-ABL1-independent TKI resistance $(5,6)$ and disease progression in CML, particularly during the progression to B lymphoid BP (26-29). In leukemia cells from patients with CML who exhibited disease progression during imatinib therapy, Hck and Lyn were revealed to be strongly expressed and/or activated (26). Correspondingly, downregulation of Lyn by siRNA induced apoptosis in BCR-ABL1 positive blasts, particularly lymphoid blasts (27). In addition, mouse models have demonstrated that Lyn, Hck and Fgr are required for the transition from CML-CP to lymphoid BP $(28,29)$. In the present case, the percentage of blasts in the BM decreased from 96.1 to $17.5 \%$ following treatment with Bosutinib. In addition, the spleen demonstrated sustained shrinkage over five months of the bosutinib treatment regimen. The reduction in leukemia load was more than would be expected just from the killing of T315I-negative blasts. Thus, it is possible that the dual Src/ABL1 kinase inhibitor, bosutinib, was able to effectively suppress Src, Lyn and Hck in the T315I-positive lymphoid BP cells, thereby additionally inducing anti-leukemia effects. It will be important for future studies to compare the sensitivity of CML cells from lymphoid $\mathrm{BP}$ vs. myeloid BP to bosutinib. To date, no such data are available $(18,21)$.

The clinical course in this report suggested that the interruption of dasatinib for any reasons during CML treatment, particularly in late- $\mathrm{CP}$ or $\mathrm{AP} / \mathrm{BC}$, may induce a resistant clone against TKIs, even if the resistant mutated clone existed in BM prior to treatment with dasatinib. Indeed, poor adherence may be the predominant reason for failure to achieve adequate molecular responses in patients treated with imatinib for $>2$ years (23). A management of adverse effects and a continuation of TKI are essential for CML treatment. The selection of an appropriate TKI for each patient with CML with individual situations during TKI treatment as well as at a diagnosis of CML is the key to the best possible outcome (30).

In conclusion, the present case represents a patient with T315I-positive CML-BP. The patient responded to bosutinib as a fourth-line treatment. Bosutinib was also useful as a pre-transplant therapy for reducing leukemia cell load. Thus, patients who are resistant or intolerant to multiple TKIs, unless the leukemia cells are uniformly T315I-positive, may benefit from a bosutinib treatment regimen.

\section{Acknowledgments}

The authors would like to thank Dr. Shinya Kimura (Department of Hematology and Oncology, Saga University) and Dr. Chiaki Nakaseko (Department of Hematology, Chiba University Hospital) for their help.

\section{References}

1. Swerdlow SH, Campo E, Harris NL, Jaffe ES, Pileri SA, Stein H, Thiele $\mathrm{J}$ and Vardiman JW: WHO Classification of Tumours of Haematopoietic and Lymphoid Tissues. 4th Edition. International Agency for Research on Cancer, Lyon, pp130-139, 2008. 
2. Wilson MB, Schreiner SJ, Choi HJ, Kamens J and Smithgall TE: Selective pyrrolo-pyrimidine inhibitors reveal a necessary role for Src family kinases in Bcr-Abl signal transduction and oncogenesis. Oncogene 21: 8075-8088, 2002.

3. Lionberger JM, Wilson MB and Smithgall TE: Transformation of myeloid leukemia cells to cytokine independence by Bcr-Abl is suppressed by kinase-defective Hck. J Biol Chem 275: 18581-18585, 2000.

4. Baccarani M, Deininger MW, Rosti G, Hochhaus A, Soverini S, Apperley JF, Cervantes F, Clark RE, Cortes JE, Guilhot F, et al: European LeukemiaNet recommendations for the management of chronic myeloid leukemia: 2013. Blood 122: 872-884, 2013.

5. O'Hare T, Zabriskie MS, Eiring AM and Deininger MW: Pushing the limits of targeted therapy in chronic myeloid leukaemia. Nat Rev Cancer 12: 513-526, 2012.

6. Eide CA and O'Hare T: Chronic myeloid leukemia: Advances in understanding disease biology and mechanisms of resistance to tyrosine kinase inhibitors. Curr Hematol Malig Rep 10: 158-166, 2015.

7. Azam M, Seeliger MA, Gray NS, Kuriyan J and Daley GQ: Activation of tyrosine kinases by mutation of the gatekeeper threonine. Nat Struct Mol Biol 15: 1109-1118, 2008.

8. Cortes JE, Kantarijian H, Shah NP, Bixby D, Mauro MJ, Flinn I, O'Hare T, Hu S, Narasimhan NI, Rivera VM, et al: Ponatinib in refractory Philadelphia chromosome-positive leukemias. N Engl J Med 367: 2075-2088, 2012.

9. Cortes JE, Kim DW, Pinilla-Ibarz J, le Coutre P, Paquette R, Chuah C, Nicolini FE, Apperley JF, Khoury HJ, Talpaz M, et al: A phase 2 trial of ponatinib in Philadelphia chromosome-positive leukemias. N Engl J Med 369: 1783-1796, 2013.

10. Redaelli S, Piazza R, Rostagno R, Magistroni V, Perini P, Marega M, Gambacorti-Passerini C and Boschelli F: Activity of bosutinib, dasatinib, and nilotinib against 18 imatinib-resistant BCR/ABL mutants. J Clin Oncol 27: 469-471, 2009.

11. Redaelli S, Mologni L, Rostagno R, Piazza R, Magistroni V, Ceccon M, Viltadi M, Flynn D and Gambacorti-Passerini C: Three novel patient-derived BCR/ABL mutants show different sensitivity to second and third generation tyrosine kinase inhibitors. Am J Hematol 87: E125-E128, 2012.

12. Barrett AJ and Ito $\mathrm{S}$ : The role of stem cell transplantation for chronic myelogenous leukemia in the 21st century. Blood 125 : 3230-3235, 2015.

13. Saussele S and Silver RT: Management of chronic myeloid leukemia in blast crisis. Ann Hematol 94 (Suppl 2): S159-S165, 2015.

14. Remsing Rix LL, Rix U, Colinge J, Hantschel O, Bennett KL, Stranzl T, Müller A, Baumgartner C, Valent P, Augustin M, et al: Global target profile of the kinase inhibitor bosutinib in primary chronic myeloid leukemia cells. Leukemia 23: 477-485, 2009.

15. Cortes JE, Kantarjian HM, Brummendorf TH, Kim DW, Turkina AG, Shen ZX, Pasquini R, Khoury HJ, Arkin S, Volkert A, et al: Safety and efficacy of bosutinib (SKI-606) in chronic phase Philadelphia chromosome-positive chronic myeloid leukemia patients with resistance or intolerance to imatinib. Blood 118: 4567-4576, 2011

16. Gambacorti-Passerini C, Brümmendorf TH, Kim DW, Turkina AG, Masszi T, Assouline S, Durrant S, Kantarjian HM, Khoury HJ, Zaritskey A, et al: Bosutinib efficacy and safety in chronic phase chronic myeloid leukemia after imatinib resistance or intolerance: Minimum 24-month follow-up. Am J Hematol 89: 732-742, 2014

17. Khoury HJ, Cortes JE, Kantarjian HM, Gambacorti-Passerini C, Baccarani M, Kim DW, Zaritskey A, Countouriotis A, Besson N, Leip E, et al: Bosutinib is active in chronic phase chronic myeloid leukemia after imatinib and dasatinib and/or nilotinib therapy failure. Blood 119: 3403-3412, 2012.
18. Kantarjian HM,Cortes JE,Kim DW, Khoury HJ,BrümmendorfTH, Porkka K, Martinelli G, Durrant S, Leip E, Kelly V, et al: Bosutinib safety and management of toxicity in leukemia patients with resistance or intolerance to imatinib and other tyrosine kinase inhibitors. Blood 123: 1309-1318, 2014.

19. Cortes JE, Kim DW, Kantarjian HM, Brümmendorf TH, Dyagil I, Griskevicius L, Malhotra H, Powell C, Gogat K, Countouriotis AM and Gambacorti-Passerini C: Bosutinib versus imatinib in newly diagnosed chronic-phase chronic myeloid leukemia: Results from the BELA trial. J Clin Oncol 30: 3486-3492, 2012.

20. Brümmendorf TH, Cortes JE, de Souza CA, Guilhot F, Duvillié L, Pavlov D, Gogat K, Countouriotis AM and Gambacorti-Passerini C: Bosutinib versus imatinib in newly diagnosed chronic-phase chronic myeloid leukaemia: Results from the 24-month follow-up of the BELA trial. Br J Haematol 168: 69-81, 2015.

21. Nakaseko C, Takahashi N, Ishizawa K, Kobayashi Y, Ohashi K, Nakagawa Y, Yamamoto K, Miyamura K, Taniwaki M, Okada M, et al: A phase 1/2 study of bosutinib in Japanese adults with Philadelphia chromosome-positive chronic myeloid leukemia. Int J Hematol 101: 154-164, 2015.

22. Strati P, Kantarjian H, Thomas D, O'Brien S, Konoplev S, Jorgensen JL, Luthra R, Abruzzo L, Jabbour E, QuintasCardama A, et al: HCVAD plus imatinib or dasatinib in lymphoid blastic phase chronic myeloid leukemia. Cancer 120: 373-380, 2014.

23. Marin D, Bazeos A, Mahon FX, Eliasson L, Milojkovic D, Bua M, Apperley JF, Szydlo R, Desai R, Kozlowski K, et al: Adherence is the critical factor for achieving molecular responses in patients with chronic myeloid leukemia who achieve complete cytogenetic responses on imatinib. J Clin Oncol 28: 2381-2388, 2010.

24. Cortes J, Lipton JH, Rea D, Digumarti R, Chuah C, Nanda N, Benichou AC, Craig AR, Michallet M, Nicolini FE, et al: Phase 2 study of subcutaneous omacetaxine mepesuccinate after TKI failure in patients with chronic-phase CML with T315I mutation. Blood 120: 2573-2580, 2012.

25. Pemovska T, Johnson E, Kontro M, Repasky GA, Chen J, Wells P, Cronin CN, McTigue M, Kallioniemi O, Porkka K, et al: Axitinib effectively inhibits BCR-ABL1(T315I) with a distinct binding conformation. Nature 519: 102-105, 2015

26. Donato NJ, Wu JY, Stapley J, Gallick G, Lin H, Arlinghaus R and Talpaz M: BCR-ABL independence and LYN kinase overexpression in chronic myelogenous leukemia cells selected for resistance to STI571. Blood 101: 690-698, 2003.

27. Ptasznik A, Nakata Y, Kalota A, Emerson SG and Gewirtz AM: Short interfering RNA (siRNA) targeting the Lyn kinase induces apoptosis in primary, and drug-resistant, BCR-ABL1(+) leukemia cells. Nat Med 10: 1187-1189, 2004.

28. Hu Y, Liu Y, Pelletier S, Buchdunger E, Warmuth M, Fabbro D, Hallek M, Van Etten RA and Li S: Requirement of Src kinases Lyn, Hck and Fgr for BCR-ABL1-induced B-lymphoblastic leukemia but not chronic myeloid leukemia. Nat Genet 36: 453-461, 2004.

29. Hu Y, Swerdlow S, Duffy TM, Weinmann R, Lee FY and Li S: Targeting multiple kinase pathways in leukemic progenitors and stem cells is essential for improved treatment of Ph+leukemia in mice. Proc Natl Acad Sci USA 103: 16870-16875, 2006.

30. Larson RA: Is there a best TKI for chronic phase CML? Blood 126: 2370-2375, 2015. 